\title{
Predictors of Change Following Cognitive-Behavioral Treatment of Children with Anxiety Problems: A Preliminary Investigation on Negative Automatic Thoughts and Anxiety Control
}

\author{
Peter Muris · Birgit Mayer · Madelon den Adel · Tamara Roos • \\ Julie van Wamelen
}

Published online: 26 July 2008

(C) The Author(s) 2008

\begin{abstract}
The purpose of the present study was to evaluate negative automatic thoughts and anxiety control as predictors of change produced by cognitive-behavioral treatment of youths with anxiety disorders. Forty-five high-anxious children aged between 9 and 12 years who were selected from the primary school population, received a standardized CBT intervention that was provided in a group format. Before and after the intervention, children completed scales of negative automatic thoughts and perceived control over anxiety-related events as well as a questionnaire for measuring DSM-defined anxiety disorders symptoms, which was the outcome measure. Results indicated that CBT was effective in reducing children's anxiety symptoms. Most importantly, the reduction of anxiety disorders symptoms was significantly associated with a decrease in negative automatic thoughts and an increase of anxiety control, which provides support for the notion that these variables are candidate mediators of CBT in anxious youths.
\end{abstract}

Keywords Anxiety · Children · Cognitive-behavioral treatment .

Negative automatic thoughts $\cdot$ Anxiety control $\cdot$ Predictors of change

\section{Introduction}

Anxiety disorders are among the most common psychiatric disorders in children and adolescents. Recent epidemiological studies show that up to 5\% of youths suffer from anxiety complaints that are severe enough to interfere with their daily functioning $[1,2]$. Specific phobia, social phobia, generalized anxiety disorder, and separation anxiety disorder are most common, with mean prevalence rates up to 5\% [3]. Research has indicated that in a substantial proportion of the youths, anxiety disorders become chronic, causing significant interference with daily functioning for many years [4]. Childhood anxiety disorders also show considerable comorbidity with other psychiatric problems, in

P. Muris $(\varangle) \cdot$ B. Mayer $\cdot$ M. den Adel $\cdot$ T. Roos $\cdot$ J. van Wamelen Institute of Psychology, Erasmus University Rotterdam, Burgemeester Oudlaan 50, Suite T13-37, P.O. Box 1738, 3000 DR Rotterdam, The Netherlands e-mail: muris@fsw.eur.nl 
particular depression. For example, Costello et al. [3] noted that depression is more than 8 times as likely in youths with anxiety disorders than in youths without anxiety disorders.

During the past two decades, considerable progress has been made with the treatment of anxiety disorders in children and adolescents. Specific cognitive-behavioral therapy (CBT) interventions have been developed, which include various techniques that are thought to be effective for reducing anxiety, including cognitive restructuring, coping self-talk, in vivo exposure, modeling, and relaxation training. In a first randomized clinical trial to evaluate the effectiveness of such an intervention in youths [5], 47 children with anxiety disorders were assigned to a CBT intervention or a waiting-list control condition. Outcome was evaluated using child self-report, parent report, teacher report, and behavioral observation. The results showed that there were significant pre- to post-treatment changes in the CBT intervention group, whereas the waiting-list control group remained relatively unchanged. More precisely, the CBT intervention resulted in substantial reductions of self-reported anxiety symptoms, parent- and teacher-reported internalizing symptoms, and observable anxious behavior. Importantly, many of the treated children (64\%) no longer met the diagnostic criteria for an anxiety disorder at post-treatment, which indicated that the observed treatment effects were also clinically significant.

Further therapy outcome studies on the efficacy of CBT in anxiety disordered youths have demonstrated that (1) this type of intervention yields statistically and clinically significant reductions of children's anxiety symptoms that are superior to those observed in waiting-list control conditions [6], (2) CBT provided in a group format is equally effective as when provided in an individual format [7, 8], (3) the effectiveness of CBT can be enhanced by incorporating family training $[9,10]$, although this has not been shown in all the studies comparing CBT with a CBT plus parent training intervention [11], and (4) the positive effects of CBT are maintained over time periods as long as 5-7 years [12, 13]. On the basis of the available evidence, it can be concluded that CBT programs have proven to be successful in treating youths with anxiety disorders [14-16].

In spite of the fact that a psychological intervention such as CBT has shown to be effective in treating children with anxiety disorders, fairly little is known about the mechanisms of action during such an intervention [17]. According to cognitive-behavioral models, negative automatic thoughts play a crucial role in the pathogenesis of anxiety disorders [18]. Kendall [19], who adapted this model to childhood anxiety, assumes that due to a chronic activity of danger schemas, information processing resources are chronically focused on threat-relevant information, which in turn yields dysfunctional and maladaptive thoughts that continuously fuel feelings of anxiety. There is indeed accumulating empirical support for this model. To begin with, the past 10 years have seen a marked rise in research on anxiety-related information processing abnormalities in children and adolescents $[20,21]$. These studies have generally demonstrated that anxious youths display biases and distortions in various stages of the processing of anxiety-related information. More precisely, there is some evidence indicating that anxious children and adolescents display an attentional bias for threat during the encoding stage, whereas there is clear support for the presence of various types of interpretation biases occurring during the more conceptual stages of information processing. Further, various studies have documented that anxious children indeed display higher levels of negative thinking. For example, Ronan et al. [22] and Schniering and Rapee [23] developed questionnaires for measuring anxiety-related negative thinking, and have shown that children with anxiety disorders score significantly higher on such measures than non-anxious control children.

All this research provides support for the idea that distorted cognition and negative thinking underlie anxiety problems in youths. However, only very little research has investigated whether change in these mechanisms indeed form the basis for an effective CBT 
intervention. One exception is a series of studies conducted by Kendall and Treadwell $[24,25]$ who assessed anxious self-statements in clinically referred youths with anxiety disorders, and then examined whether change in such self-statements was predictive of change in anxiety symptomatology as produced by CBT. The results indeed demonstrated that changes in anxious self-statements mediated treatment gains, which of course is in keeping with the cognitive-behavioral model of anxiety disorders [26, 27]. At the same time, it is important to note that the observed cognitive changes explained a fairly small proportion of the variance in the effects of CBT, and hence should only be considered as partial mediators of such an intervention. As mentioned earlier, CBT interventions for childhood anxiety typically contain more elements than cognitive restructuring alone, and so it seems plausible that other mechanisms are also at work. One obvious candidate in this regard seems to be perceived control. Previous theoretical work by Chorpita and Barlow [28] has proposed that low levels of perceived control are an essential feature of anxiety pathology in youths. Empirical support for this idea comes from a study by Weems et al. [29] who administered a questionnaire for measuring perceptions of control over anxiety-related events to children and adolescents with anxiety disorders and non-anxious controls. The results clearly indicated that anxious children and adolescents displayed lower levels of perceived control over anxiety-related events than their non-anxious counterparts, which made the authors conclude that "anxiety disorders in youths are associated with beliefs that anxiety is uncontrollable" (p. 557). During CBT children are taught various techniques for coping more adequately with their anxious cognitions and feelings, including cognitive restructuring, positive selftalk, problem-focused coping (i.e., approach instead of avoidance behavior), relaxation, and self-reinforcement, and as such it is well conceivable that perceptions of control over anxietyrelated events are significantly enhanced by such an intervention.

The present study further explored potential predictors of treatment change after CBT for children with anxiety disorders. Following Kendall and Treadwell [24, 25], we evaluated the role of negative thinking, and in particular anxiety-related negative automatic thoughts, as a possible correlate of treatment gains. In addition, we investigated whether perceived control over anxiety-related events represents an alternative correlate of treatment effects produced by such an intervention. For this purpose, a sample of selected school children who displayed high levels of anxiety disorders symptoms were treated with a manualized CBT intervention that was provided in a group format. Before and after treatment, children completed scales of negative automatic thoughts and perceived control over anxiety-related events, which were the hypothesized predictors of treatment change, as well as a questionnaire for measuring anxiety disorders symptoms as defined by the Diagnostic and Statistical Manual of Mental Disorders (DSM) [30], which was the treatment outcome measure. It was hypothesized that treatment effects as indexed by reductions in anxiety disorders symptoms are associated with a decrease in negative automatic thoughts and an increase in perceived control over anxietyrelated events. In addition, by performing regression analyses in which both predictors were entered simultaneously it was also possible to examine their relative importance in the prediction of treatment effects of CBT in anxious youths.

\section{Method}

Participants

Children were recruited from five regular primary schools in Zuid-Holland (Dordrecht and Rotterdam) and Zeeland (Zierikzee), The Netherlands. After obtaining informed consent 
from their parents, 178 9- to 12-year-old children (86 boys and 92 girls) completed the Revised version of the Screen for Child Anxiety Related Emotional Disorders (SCARED-R) [31], which is a standardized and well-validated self-report questionnaire for measuring DSM-defined anxiety disorders symptoms. Fifty-five children (29 boys and 26 girls) were selected from this sample because they displayed scores in the top $10 \%$ of the social phobia, separation anxiety disorder, and/or generalized anxiety disorder subscales of the SCARED-R. The parents of 45 children ( 23 boys and 22 girls; mean age $=10.33$ years, $S D=1.04)$ agreed that their child would participate in the treatment program. These children scored in the top $10 \%$ of social phobia $(n=22)$, separation anxiety disorder $(n=18)$, and generalized anxiety disorder $(n=27)$. Most children $(n=30)$ scored high on only one of these anxiety disorders, 8 children exhibited high scores on two of them, whereas 7 children displayed high scores on all three anxiety disorders.

\section{Assessment}

The SCARED- $R$ is an extension of the original SCARED [32, 33] and measures symptoms of the entire spectrum of anxiety disorders that according to the DSM may occur in children and adolescents. Thus, the scale consists of 69 items measuring symptoms of separation anxiety disorder (8 items; e.g., "I don't like being away from my family"), generalized anxiety disorder (9 items; e.g., "I worry about things working out for me"), panic disorder (13 items; e.g., "When frightened, my heart beats fast"), obsessive-compulsive disorder ( 9 items; e.g., "I have thoughts that frighten me"), traumatic stress disorder (4 items; e.g., "I have frightening dreams about a very aversive event"), social phobia (7 items; e.g., "I am shy with people I don't know well”), specific phobia (15 items; e.g., "I am afraid of the dentist"), and school phobia (4 items; e.g., "I am scared to go to school"). Children and adolescents have to indicate how frequently they experience each symptom on a three-point scale: $0=$ almost never, $1=$ sometimes, or $2=$ often. SCARED-R total and subscale scores can be obtained by summing across relevant items, with higher scores reflecting higher levels of anxiety symptoms. Previous studies have shown that the SCARED-R possesses adequate internal consistency [34, 35], sufficient test-retest stability [36], and acceptable validity, that is, the scale correlates substantially with other childhood anxiety measures $[37,38]$ and discriminates between children with and without anxiety disorders [35, 39, 40]. Thus, the SCARED-R seems to be a reliable and valid questionnaire for assessing symptoms of anxiety disorders in youths.

Anxiety-related negative automatic thoughts were assessed with the Children's Automatic Thoughts Scale (CATS) [23], which contains 40 items referring to negative beliefs in the domains of social threat (10 items; e.g., "Kids will think that I am stupid"), physical threat (10 items; e.g., "I am going to have an accident"), personal failure (10 items; e.g., "I can't do anything right"), and hostility (10 items; e.g., "I have the right to take revenge on people if they deserve it"). Children have to indicate the frequency with which they have experienced various thoughts during the past week by means of 5-point scales ranging from $0=$ not at all to $4=$ all the time. Scores on items are summed to yield a score for each domain and a total score for the full scale. In the present study, only the social threat and physical threat domains were used, because these two domains are especially indicative for anxiety problems [23, 41]. Psychometric evaluations of the CATS have demonstrated that the scale is reliable in terms of internal consistency and test-retest stability [23], has a clear-cut four-factor structure representing the four domains of 
negative automatic thoughts [42], correlates positively with measures of internalizing and externalizing psychopathology, and differentiates reasonably well between children and adolescents with various types of psychological disorders [23, 43].

A shortened 13-item version of the Anxiety Control Questionnaire for Children (ACQC) [29] was employed to measure children's perception of control over external threats (e.g., "When something scares me, there is always something I can do") and internal, emotional, and bodily responses associated with anxiety (e.g., "I can usually stop thinking about things that make me nervous or afraid if I try"). Given the relatively young age of the children in this study, we selected those items from the original ACQ-C that (a) were easy to read and comprehend, (b) did not contain a 'double negation', and (c) clearly referred to the control of fear and anxiety rather than the control of negative emotion in general. Children are asked how much they agree with each of the items using a Likert scale with $0=$ not at all and $4=$ very much. A total score (range $0-52$ ) was obtained by summing across all items, with a higher score being indicative for higher levels of perceived control over anxiety-related events (or more briefly 'anxiety control'). Tentative support has emerged for the reliability and validity of the ACQ-C. That is, the scale has been found to possess good internal consistency, and correlates in a theoretically meaningful way with other scales of control and measures of anxiety [29, 44]. Further, as mentioned in the introduction, ACQ-C scores distinguished youths with anxiety disorders from youths without such problems [29].

\section{Cognitive-Behavioral Treatment}

The Coping Koala CBT program [45] is the Australian adaptation of Kendall's Coping Cat program [46]. Briefly, this manualized treatment program that can be delivered in a group format, and consists of 12 sessions of about 30 min which focus on recognizing anxious feelings and somatic reactions to anxiety, cognitive restructuring in anxietyprovoking situations, coping self-talk, exposure to feared stimuli, evaluating performance, and administering self-reinforcement. During the first four sessions, anxiety management procedures (e.g., relaxation) are introduced, role-played by the therapist and practiced by the children. Throughout the remaining eight sessions children practice to master their anxiety and fears by means of the so-called FEAR-plan, in which children learn to use physiological, cognitive, and behavioral coping strategies in increasingly threatening situations: $\mathrm{F}=$ feeling good by learning to relax, $\mathrm{E}=$ expecting good things to happen through positive self-talk, $\mathrm{A}=$ actions to take when confronted with fear stimuli, and $\mathrm{R}=$ rewarding oneself for efforts to overcome fear and worry. Each child applies the FEAR-plan exercises in idiosyncratic anxietyprovoking situations, so that the intervention program is tailored to individual children within the group.

\section{Procedure}

After obtaining consent from the schools and children's parents, a research assistant administered the SCARED-R, CATS and ACQ-C during classroom sessions. As mentioned earlier, the SCARED-R was employed to select high-anxious children from the school populations. Gender-appropriate cut-off scores [31] were used to identify boys and girls scoring in the top $10 \%$ of the social phobia, separation anxiety disorder, and generalized anxiety disorder subscales. The focus was on these specific anxiety disorders 
because the Coping Koala CBT program is generally considered as appropriate for treating these types of anxiety problems [47]. Selected children and their parents were approached for a second time to provide written informed consent for the intervention part of the study. Those who decided to participate were assigned to small groups of 3-6 children within one and the same school. The CBT program was run by three trained master students in clinical psychology who visited the schools during 6 weeks to provide $1 \mathrm{~h}$ treatment sessions (in which two of the original sessions of the Coping Koala were combined). Each week, an experienced cognitive-behavioral therapist/child psychologist supervised the master students. During this supervision, upcoming sessions were carefully prepared and specific treatment issues were discussed. Within 1 week after the last treatment session, a research assistant (who had not been involved in the intervention of the children) visited the schools and asked the participating children to complete the SCARED-R, CATS, and ACQ-C for a second time. On the basis of the scores on the post-treatment assessment, children and parents were contacted again and eventually referred to clinical treatment facilities in case the treatment had not yielded sufficient effect.

\section{Statistical Analysis}

Paired $t$-tests were carried out to examine pre- to post-treatment changes in anxiety disorders symptoms, negative automatic thoughts, and perceived control over anxiety-related events. Cohen's $d$ [48] was computed to get an impression of the effect size of the treatment effects. To examine the relation between changes in negative automatic thoughts and perceived control over anxiety-related events and reductions in anxiety symptoms produced by treatment, correlations and regression analyses were performed. In the regression analyses, pre- to post-treatment change scores of negative automatic thoughts and anxiety control were entered as predictors of change scores of anxiety disorders symptoms, which served as the dependent variable. This procedure made it possible to examine the relative contributions of both variables in explaining the effects of CBT on anxiety disorders symptoms.

\section{Results}

General Results

Before addressing the main research issues, a number of general findings will be discussed. First of all, the mean SCARED-R score of the total school sample was 28.69 $(S D=20.72)$, which is somewhat lower that the normative mean score reported by Muris et al. [31]. Nevertheless, the mean pre-treatment score of the selected high-anxious children $(M=48.44, S D=19.48)$ comes close to the mean SCARED-R score as obtained in a clinically referred sample of youths with anxiety disorders $(M=51.20, S D=21.30)$ [35]. This suggests that the selected children displayed fairly high levels of anxiety disorders symptoms. Second, the questionnaires that were used in this study all demonstrated good reliability: Cronbach's alphas were .96 for the SCARED-R total score, .80 for social phobia, .80 for separation anxiety disorder, .83 for generalized anxiety disorder, .93 for the CATS, and .89 for the ACQ-C. Third and finally, correlations among anxiety disorders symptoms on the one hand, and negative automatic thoughts and anxiety control on the other hand, were as expected. That is, anxiety symptoms correlated positively with 
negative automatic thoughts [all $r(N=178)$ s between .40 (social phobia) and .68 (generalized anxiety disorder), $p \mathrm{~s}<.001$ ], whereas such symptoms were negatively linked to anxiety control [all $r(N=178)$ s between -.25 (social phobia) and -.36 (SCARED-R total score), $p s<.01]$. In other words, higher levels of anxiety disorders symptoms were associated with higher levels of negative automatic thoughts and lower levels of perceived control over anxiety-related events.

\section{Treatment Effects}

Table 1 displays mean pre-treatment and post-treatment scores on various questionnaires. Paired $t$-tests demonstrated that anxiety disorders symptoms significantly decreased from pre- to post-treatment [all $t(44) \mathrm{s} \geq 3.92, p \mathrm{~s}<.001$ ]. Inspection of the effect sizes (Cohen's $d$ ) revealed that these changes reflected medium (social phobia) to large effects. Pre- to post-treatment changes in negative automatic thoughts and anxiety control were also significant $[t(44)$ s being $2.37, p<.05$ and $-2.98, p<.01$, respectively]. As shown in Table 1, negative automatic thoughts decreased, while anxiety control increased after the CBT intervention. In both cases, these changes were indicative of a medium effect size.

\section{Predictors of Treatment Gains}

Correlations were computed to examine whether reductions in anxiety disorders symptoms were associated with a decrease in negative automatic thoughts and an increase in perceived control. The results demonstrated that change scores for anxiety symptoms as indexed by pre-treatment minus post-treatment SCARED-R total scores were indeed significantly related to change scores for negative automatic thoughts $(r=.54, p<.001)$. Further correlations showed that this appeared particularly true for symptoms of separation anxiety disorder $(r=.47, p<.01)$ and generalized anxiety disorder $(r=.42, p<.01)$, but less so for symptoms of social phobia $(r=.28, p>.05)$.

Changes in total SCARED-R scores were not significantly associated with change scores in anxiety control $(r=-.21, p>.10)$. However, when computing correlations for separate types of symptoms, results indicated that change scores in social phobia and

Table 1 Mean scores (standard deviations) on various questionnaires at pre-treatment and post-treatment

\begin{tabular}{lcccc}
\hline & Pre-treatment & Post-treatment & $t(44)$ & Cohen's $d$ \\
\hline $\begin{array}{l}\text { SCARED-R } \\
\quad \text { Total score }\end{array}$ & $48.44(19.48)$ & $34.40(18.67)$ & $4.79 * * *$ & .74 \\
$\quad$ Social phobia & $7.31(3.50)$ & $5.64(3.59)$ & $3.92 * * *$ & .47 \\
$\quad$ Separation anxiety disorder & $5.91(3.75)$ & $3.73(3.14)$ & $4.64 * * *$ & .63 \\
$\quad$ Generalized anxiety disorder & $7.29(3.76)$ & $4.49(2.29)$ & $5.45 * * *$ & .90 \\
CATS & $18.91(12.50)$ & $14.36(11.82)$ & $2.37 *$ & .37 \\
$\quad$ Negative automatic thoughts & & & & \\
ACQ-C & $21.40(8.80)$ & $25.16(9.52)$ & $-2.98 * *$ & .41 \\
$\quad$ Anxiety control & & & & \\
\hline
\end{tabular}

Note: $N=45$. SCARED-R = Screen for child anxiety related emotional disorders-revised, CATS = Children's automatic thoughts scale, ACQ-C = Anxiety control questionnaire for children. $* p<.05$; $* * p<.01 ; * * * p<.001$ 
Table 2 Main results of the regression analyses in which changes in negative automatic thoughts and anxiety control predicted change in anxiety disorders symptoms

\begin{tabular}{|c|c|c|c|c|}
\hline & $B$ & $S E$ & $\beta$ & $R^{2}$ \\
\hline$\Delta$ SCARED-R total anxiety score & & & & $.30 * *$ \\
\hline$\Delta$ CATS negative automatic thoughts & .79 & .20 & $.52 * * *$ & \\
\hline$\Delta \mathrm{ACQ}-\mathrm{C}$ anxiety control & -.23 & .31 & -.10 & \\
\hline$\Delta$ SCARED-R social phobia & & & & $.17 *$ \\
\hline$\Delta$ CATS negative automatic thoughts & .05 & .03 & .21 & \\
\hline$\Delta$ ACQ-C anxiety control & -.11 & .05 & $-.31 *$ & \\
\hline$\Delta$ SCARED-R separation anxiety disorder & & & & $.23 * *$ \\
\hline$\Delta$ CATS negative automatic thoughts & .11 & .03 & $.44 * *$ & \\
\hline$\Delta \mathrm{ACQ}-\mathrm{C}$ anxiety control & -.04 & .05 & -.12 & \\
\hline$\Delta$ SCARED-R generalized anxiety disorder & & & & $.26^{* *}$ \\
\hline$\Delta$ CATS negative automatic thoughts & .09 & .04 & $.35 *$ & \\
\hline$\Delta \mathrm{ACQ}-\mathrm{C}$ anxiety control & -.13 & .06 & $-.31 *$ & \\
\hline
\end{tabular}

Note: $N=45$. SCARED-R $=$ Screen for child anxiety related emotional disorders-revised, CATS $=$ Children's automatic thoughts scale, ACQ-C = Anxiety control questionnaire for children

$* p<.05 ; * * p<.01 ; * * * p<.001$

generalized anxiety disorder (but not separation anxiety disorder) were significantly linked to the change score in anxiety control ( $r$ s being $-.36, p<.05$ and $-.39, p<.01$, respectively). In other words, decreases of these types of anxiety symptoms were accompanied by an increase in anxiety control.

The correlation between change scores in negative automatic thoughts and change scores in anxiety control was non-significant $(r=-.23, p>.10)$, which indicated that both variables operated relatively independent of each other.

Regression analyses were performed to examine the relative importance of (changes in) negative automatic thoughts and anxiety control in the prediction of treatment effects of CBT. As can be seen in Table 2, the regression analysis predicting change in SCARED-R total scores indicated that only change in negative automatic thoughts accounted for a significant proportion of the variance $(\beta=.52, p<.001)$. When predicting the separate types of anxiety disorders symptoms, the picture was somewhat different. Results indicated that change in anxiety control was a unique predictor of change in symptoms of social phobia, change in negative automatic thoughts was a independent predictor of change in symptoms of separation anxiety disorder, whereas in the regression analysis predicting change in symptoms of generalized anxiety disorder both variables accounted for a unique and significant proportion of the variance. Note that the changes in negative automatic thoughts and anxiety control explained between $17 \%$ and $30 \%$ of the variance in change of anxiety disorders symptoms from pre- to post-treatment.

\section{Discussion}

In the present study a sample of selected high-anxious children were treated with a standardized CBT program with the purpose of exploring whether changes in negative automatic thoughts and anxiety control are predictors of the reduction in anxiety produced 
by such an intervention. The main results can be catalogued as follows. First, CBT resulted in a significant decrease of children's anxiety disorders symptoms. This finding is in keeping with previous research showing that this type of intervention is fairly effective for reducing anxiety problems in youths [5-10]. In passing, it should be noted that the pre- to post-treatment changes in anxiety symptoms as observed in the present study had an effect size (Cohen's $d=.74$ ) that comes close to that reported by In-Albon and Schneider [15] in their meta-analysis on the efficacy of cognitive-behavioral interventions for anxious youths (Cohen's $d=.86$ ). Second, the CBT intervention also produced the hypothesized changes in negative automatic thoughts and anxiety control. More precisely, negative automatic thoughts significantly decreased from pre- to post-treatment, whereas perceptions of anxiety control significantly increased. Third and most importantly, changes in anxiety disorders symptoms were associated with changes in negative automatic thoughts and anxiety control, which is in keeping with the idea that these variables are indeed candidate mediators of the treatment effects produced by CBT.

A number of remarks can be made with regard to the finding that negative automatic thoughts and anxiety control were correlated with outcome of CBT. To begin with, change scores in negative automatic thoughts and change scores in anxiety control were not significantly associated, and so it was not surprising that the relations between both variables and treatment outcome did hardly change in the regression analyses which controlled for the shared variance among them. In other words, negative automatic thoughts and anxiety control were found to make fairly unique contributions to treatment outcome. Further, the influence of these variables appeared to differ across various types of anxiety disorders symptoms. That is, although the data indicated that the effects of CBT are generally associated with changes in negative automatic thoughts [24, 25], results also suggested that an increase in perceived control over anxiety-related events was (also) linked to treatment change in symptoms of social phobia and generalized anxiety disorder. It is tempting to use these findings as a basis for speculating on the pathological processes underlying various types of anxiety symptoms in youths. For example, one could argue that negative automatic thoughts play a more dominant role in symptoms of separation anxiety disorder, low perceived control over anxiety is more important in symptoms of social phobia, whereas both mechanisms seem to be at work in symptoms of generalized anxiety disorder. Of course, more empirical work is necessary to further investigate these ideas. Finally, change in negative automatic thoughts and anxiety control accounted for at best $30 \%$ of the variance in the effects of treatment. This percentage of explained variance is comparable to that reported by Kendall and Treadwell [24] and seems to warrant the conclusion that there are still other factors involved that may contribute to the therapeutic change as a result of CBT. Besides the so-called nonspecific effects of psychological treatment (e.g., the quality of the client-therapist relationship) [49], these other factors may involve correction of cognitive biases, increased use of adaptive coping, and reduction of physiological arousal [17, 50].

It should be admitted that the present study suffers from various limitations. A first limitation pertains to the fact that we did not include a no-treatment control condition in this study. In the absence of such a control condition, one cannot determine whether the observed changes in anxiety symptoms, automatic thoughts, and anxiety control were due to treatment or simply a function of the passage of time or other variables present in the lives of these children. However, many studies have already shown that CBT is more effective than a waiting list (e.g., [5, 6]) and similar research in high-anxious school populations has demonstrated that anxiety problems do not remit spontaneously [51-53]. A second shortcoming was that all questionnaires were completed by children themselves, 
thereby introducing a shared variance problem which raises questions about the validity of the observed findings. As noted by Weersing and Weisz [17], the present study certainly is no exception in this respect, but as these authors rightly point out future studies may benefit from performance-based measures to index changes in anxiety symptomatology and related phenomena [54]. A third demerit is concerned with the fairly small sample size of the present study $(N=45)$, which may have resulted in limited statistical power. For example, one could argue that some of the observed relationships would have attained statistical significance in case we had included more children in this study. A fourth deficiency has to do with the given that we relied on a non-clinical sample of children who were selected on the basis of their (high) scores on an anxiety questionnaire. Thus, it remains to be seen whether the current findings can be generalized to clinically referred children who suffer from severe and persistent anxiety disorders. A fifth limitation applies to the fairly young age of the children included in this study (i.e., 9-12 years). As developmental factors (e.g., cognitive maturation) are thought to have an impact on anxiety in youths [47], it may well be that the predictors of change produced by CBT vary as a function of children's age. A sixth deficiency pertains to the fact that no formal treatment integrity check was conducted. Note, however, that the Coping Koala is a fully manualized treatment program that provides a detailed description of the exercises and tasks that children have to perform during each of the sessions. In addition, upcoming sessions were carefully prepared under the supervision of an experienced cognitive-behavioral therapist who also checked whether the students-therapists closely adhered to the program. A seventh and final shortcoming is concerned with the fact that we did not include a follow-up assessment in our study. Although it is already known that CBT programs such as the Coping Koala are also highly effective in the long run $[12,13]$, such follow-up data could have provided important information on the durability of changes in anxiety symptoms and the predictor variables.

\section{Summary}

The current investigation examined negative automatic thoughts and anxiety control as predictors of change produced by cognitive-behavioral treatment of anxious youths. Despite various limitations, it was shown that the reduction of anxiety disorders symptoms observed after a CBT intervention was significantly associated with a decrease in negative automatic thoughts as well as an increase of anxiety control. This is nicely in keeping with the notion that anxiety disorders in youths are the result of various etiological factors [55] and justifies that a CBT intervention such as the Coping Koala (or the Coping Cat) includes a range of techniques which all aim at soothing different aspects of this type of psychopathology [47]. Future studies should make a real attempt to investigate negative automatic thoughts and anxiety control as potential mediators of CBT. Several authors [56, 57] have outlined the methodology for examining mediating variables of treatments effects. To establish whether a variable acts as a mediator of the intervention, the inclusion of a notreatment control condition is a prerequisite so that it becomes possible to study the links between treatment status (CBT vs. waitlist), the presumed mediator, and the outcome variable (i.e., change in anxiety). Further, researchers should try to assess the mediator variable multiple times during the course of the intervention, to really establish whether change in the mediator precedes change in symptoms. Research including various candidate mediators could yield a better understanding of the crucial variables that produce therapeutic change in $\mathrm{CBT}$ and may also make it possible to link various treatment components to specific mediators. 
Acknowledgments Children, parents, and staffs of primary schools "Statenschool" and "J.F. Kennedy school" in Dordrecht, "Ds. J.J. Buskesschool" in Rotterdam, and "Johan Louis de Jonge school" and "Jan Wouter van den Doel school" in Zierikzee are kindly thanked for their participation in the present study.

Open Access This article is distributed under the terms of the Creative Commons Attribution Noncommercial License which permits any noncommercial use, distribution, and reproduction in any medium, provided the original author(s) and source are credited.

\section{References}

1. Costello EJ, Mustillo S, Erkanli A, Keeler G, Angold A (2003) Prevalence and development of psychiatric disorders in childhood and adolescence. Arch Gen Psychiatry 60:837-844. doi:10.1001/ archpsyc.60.8.837

2. Ford T, Goodman R, Meltzer H (2003) The British child and adolescent mental health survey 1999: the prevalence of DSM-IV disorders. J Am Acad Child Adolesc Psychiatry 42:1203-1211. doi:10.1097/ 00004583-200310000-00011

3. Costello EJ, Egger HL, Angold A (2004) Developmental epidemiology of anxiety disorders. In: Ollendick TH, March JS (eds) Phobic and anxiety disorders in children and adolescents. A clinician's guide to effective psychosocial and pharmacological interventions. Oxford University Press, New York, pp 61-91

4. Kessler RC, Berglund P, Demler O, Jin R, Merikangas KR, Walters EE (2005) Lifetime prevalence and age-of-onset distributions of DSM-IV disorders in the national comorbidity survey replication. Arch Gen Psychiatry 62:593-602. doi:10.1001/archpsyc.62.6.593

5. Kendall PC (1994) Treating anxiety disorders in children: results of a randomized clinical trial. J Consult Clin Psychol 62:100-110. doi:10.1037/0022-006X.62.1.100

6. Kendall PC, Flannery-Schroeder E, Panicelli-Mindel S, Southam-Gerow M, Henin A, Warman M (1997) Therapy for youths with anxiety disorders: a second randomized clinical trial. J Consult Clin Psychol 65:366-380. doi:10.1037/0022-006X.65.3.366

7. Flannery-Schroeder E, Kendall PC (2000) Group and individual cognitive-behavioral treatments for youth with anxiety disorders: a randomized clinical trial. Cognit Ther Res 24:251-278. doi: 10.1023/A:1005500219286

8. Silverman WK, Kurtiness WM, Ginsburg GS, Weems CF, Rabian B, Serafini LT (1999) Contingency management, self-control, and education support in the treatment of childhood phobic disorders: a randomized clinical trial. J Consult Clin Psychol 67:675-687. doi:10.1037/0022-006X.67.5.675

9. Barrett PM (1998) Evaluation of cognitive-behavioral group treatments for childhood anxiety disorders. J Clin Child Psychol 27:459-468. doi:10.1207/s15374424jccp2704_10

10. Barrett PM, Dadds MR, Rapee RM (1996) Family treatment of childhood anxiety: a controlled trial. J Consult Clin Psychol 64:333-342. doi:10.1037/0022-006X.64.2.333

11. Barmish AJ, Kendall PC (2005) Should parents be co-clients in cognitive-behavioral therapy for anxious youth? J Clin Child Adolesc Psychol 34:569-581. doi:10.1207/s15374424jccp3403_12

12. Barrett PM, Duffy AL, Dadds MR, Rapee RM (2001) Cognitive-behavioral treatment of anxiety disorders in children: long-term (6-year) follow-up. J Consult Clin Psychol 69:135-141. doi: 10.1037/0022-006X.69.1.135

13. Kendall PC, Safford S, Flannery-Schroeder E, Webb A (2004) Child anxiety treatment: outcomes in adolescence and impact on substance use and depression at 7.4-year follow-up. J Consult Clin Psychol 72:276-287. doi:10.1037/0022-006X.72.2.276

14. Cartwright-Hatton S, Roberts C, Chitsabesan P, Fothergill C, Harrington R (2004) Systematic review of the efficacy of cognitive behaviour therapies for childhood and adolescent anxiety disorders. Br J Clin Psychol 43:421-436. doi:10.1348/0144665042388928

15. In-Albon T, Schneider S (2007) Psychotherapy of childhood anxiety disorders: a meta-analysis. Psychother Psychosom 76:15-24. doi:10.1159/000096361

16. James A, Soler A, Weatherall R (2005) Cognitive behavioural therapy for anxiety disorders in children and adolescents. Cochrane Database Syst Rev 4:CD004690

17. Weersing VR, Weisz JR (2002) Mechanisms of action in youth psychotherapy. J Child Psychol Psychiatry 43:3-29. doi:10.1111/1469-7610.00002

18. Beck AT, Emery G, Greenberg RL (1985) Anxiety disorders and phobias: a cognitive perspective. Basic Books, New York

19. Kendall PC (1985) Toward a cognitive-behavioral model of child psychopathology and a critique of related interventions. J Abnorm Child Psychol 13:357-372. doi:10.1007/BF00912722 
20. Vasey MW, MacLeod C (2001) Information-processing factors in childhood anxiety: a review and developmental perspective. In: Vasey MW, Dadds MR (eds) The developmental psychopathology of anxiety. Oxford University Press, New York, pp 253-277

21. Muris P, Field A (2008) Distorted cognition and pathological anxiety in children and adolescents. Cogn Emot 22:395-421. doi:10.1080/02699930701843450

22. Ronan KR, Kendall PC, Rowe M (1994) Negative affectivity in children: development and validation of a self-statement questionnaire. Cognit Ther Res 18:509-528. doi:10.1007/BF02355666

23. Schniering CA, Rapee RM (2002) Development and validation of a measure of children's automatic thoughts: the children's automatic thoughts scale. Behav Res Ther 40:1091-1109. doi:10.1016/S00057967(02)00022-0

24. Kendall PC, Treadwell K (2007) The role of self-statement as a mediator in treatment for youth with anxiety disorders. J Consult Clin Psychol 75:380-389. doi:10.1037/0022-006X.75.3.380

25. Treadwell K, Kendall PC (1996) Self-talk in youth with anxiety disorders: states of mind, content specificity, and treatment outcome. J Consult Clin Psychol 64:941-950. doi:10.1037/0022-006X.64.5.941

26. Kendall PC, Chansky TE (1991) Considering cognition in anxiety-disordered children. J Anxiety Disord 5:167-185. doi:10.1016/0887-6185(91)90027-Q

27. Kendall PC, MacDonald JP (1993) Cognition in the psychopathology of youth and implications for treatment. In: Dobson KS, Kendall PC (eds) Psychopathology and cognition. Academic Press, San Diego, CA, pp 387-427

28. Chorpita BF, Barlow DH (1998) The development of anxiety: the role of control in the early environment. Psychol Bull 124:3-21. doi:10.1037/0033-2909.124.1.3

29. Weems CF, Silverman WK, Rapee RM, Pina AA (2003) The role of control in childhood anxiety disorders. Cognit Ther Res 27:557-568. doi:10.1023/A:1026307121386

30. American Psychiatric Association (2000) Diagnostic and statistical manual of mental disorders, fourth edition-text revision (DSM-IV-TR). American Psychiatric Association, Washington, DC

31. Muris P, Bodden D, Hale W, Birmaher B, Mayer B (2007) SCARED-NL. Vragenlijst over angst en bang-zijn bij kinderen en adolescenten. Boom, Amsterdam

32. Birmaher B, Brent D, Chiappetta L, Bridge J, Monga S, Baugher M (1999) Psychometric properties of the screen for child anxiety related emotional disorders (SCARED): a replication study. J Am Acad Child Adolesc Psychiatry 38:1230-1236. doi:10.1097/00004583-199910000-00011

33. Birmaher B, Khetarpal S, Brent D, Cully M, Balach L, Kaufman J et al (1997) The screen for child anxiety related emotional disorders (SCARED): scale construction and psychometric characteristics. J Am Acad Child Adolesc Psychiatry 36:545-553. doi:10.1097/00004583-199704000-00018

34. Muris P, Merckelbach H, Schmidt H, Mayer B (1999) The revised version of the screen for child anxiety related emotional disorders (SCARED-R): factor structure in normal children. Pers Individ Dif 26:99-112. doi:10.1016/S0191-8869(98)00130-5

35. Muris P, Dreessen L, Bögels SM, Weckx M, Van Melick M (2004) A questionnaire for screening a broad range of DSM-defined anxiety disorder symptoms in clinically referred children and adolescents. J Child Psychol Psychiatry 45:813-820. doi:10.1111/j.1469-7610.2004.00274.x

36. Muris P, Merckelbach H, Van Brakel A, Mayer B (1999) The revised version of the screen for child anxiety related emotional disorders (SCARED-R): further evidence for its reliability and validity. Anxiety Stress Coping 12:411-425. doi:10.1080/10615809908249319

37. Muris P, Merckelbach H, Mayer B, Van Brakel A, Thissen S, Moulaert V et al (1998) The screen for child anxiety related emotional disorders and its relationship to traditional childhood anxiety measures. J Behav Ther Exp Psychiatry 29:327-339. doi:10.1016/S0005-7916(98)00023-8

38. Muris P, Schmidt H, Merckelbach H (2000) Correlations among two self-report questionnaires for measuring DSM-defined anxiety disorder symptoms in children: the screen for child anxiety related emotional disorders and the spence children's anxiety scale. Pers Individ Dif 28:333-346. doi: 10.1016/S0191-8869(99)00102-6

39. Muris P, Merckelbach H, Mayer B, Prins E (2000) How serious are common childhood fears? Behav Res Ther 38:217-228. doi:10.1016/S0005-7967(98)00204-6

40. Muris P, Steerneman P (2001) The revised version of the screen for child anxiety related emotional disorders (SCARED-R): first evidence for its reliability and validity in a clinical sample. Br J Clin Psychol 40:35-44. doi:10.1348/014466501163463

41. Schniering CA, Rapee RM (2004) The structure of negative self-statements in children and adolescents: a confirmatory factor-analytic approach. J Abnorm Child Psychol 32:95-109. doi:10.1023/B:JA CP.0000007583.90038.7a

42. Schniering CA, Rapee RM (2004) The relationship between automatic thoughts and negative emotions in children and adolescents: a test of the cognitive content-specificity hypothesis. J Abnorm Psychol 113:464-470. doi:10.1037/0021-843X.113.3.464 
43. Schniering CA, Lyneham HJ (2007) The children's automatic thoughts scale in a clinical sample: psychometric properties and clinical utility. Behav Res Ther 45:1931-1940. doi:10.1016/j.brat.2006. 09.009

44. Weems CF, Costa NM, Watts SE, Taylor LK, Cannon MF (2007) Cognitive errors, anxiety sensitivity, and anxiety control beliefs. Their unique and specific associations with childhood anxiety symptoms. Behav Modif 31:174-201. doi:10.1177/0145445506297016

45. Heard PM, Dadds MR, Rapee RM (1991) The Coping Koala workbook. Modified Australian version of Kendall's treatment program for anxious children. University of Queensland, Brisbane, Australia

46. Kendall PC (1990) Coping Cat workbook. Temple University, Philadelphia, PA

47. Barrett PM (2001) Current issues in the treatment of childhood anxiety. In: Vasey MW, Dadds MR (eds) The developmental psychopathology of anxiety. Oxford University Press, New York, pp 304-324

48. Cohen J (1988) Statistical power analysis for the behavioral sciences, 2nd edn. Lawrence Earlbaum Associates, Hillsdale, NJ

49. Creed TA, Kendall PC (2005) Therapist alliance-building behavior within a cognitive-behavioral treatment for anxiety in youth. J Consult Clin Psychol 73:498-505. doi:10.1037/0022-006X.73.3.498

50. Prins P, Ollendick TH (2003) Cognitive change and enhanced coping: missing mediational links in cognitive behavior therapy with anxiety disordered children. Clin Child Fam Psychol Rev 6:87-105. doi:10.1023/A:1023730526716

51. Dadds MR, Spence SH, Holland DE, Barrett PM, Laurens KR (1997) Prevention and early intervention for childhood anxiety disorders: a controlled trial. J Consult Clin Psychol 65:627-635. doi: 10.1037/0022-006X.65.4.627

52. Muris P, Mayer B, Bartelds E, Tierney S, Bogie N (2001) The revised version of the screen for child anxiety related emotional disorders (SCARED-R): treatment sensitivity in an early intervention trial for childhood anxiety disorders. Br J Clin Psychol 40:323-336. doi:10.1348/014466501163724

53. Muris P, Meesters C, Van Melick M (2002) Treatment of childhood anxiety disorders: a preliminary comparison between cognitive-behavioral group therapy and a psychological placebo intervention. J Behav Ther Exp Psychiatry 33:143-158. doi:10.1016/S0005-7916(02)00025-3

54. Vasey MW, Lonigan CJ (2000) Considering the clinical utility of performance-based measures of childhood anxiety. J Clin Child Psychol 29:493-508. doi:10.1207/S15374424JCCP2904_4

55. Muris P (2007) Normal and abnormal fear and anxiety in children and adolescents. Elsevier, Oxford

56. Holmbeck GN (2002) Post-hoc probing of significant moderational and meditational effects in studies of pediatric populations. J Pediatr Psychol 27:87-96. doi:10.1093/jpepsy/27.1.87

57. Kraemer HC, Wilson GT, Fairburn CG, Agras WS (2002) Mediators and moderators of treatment effects in randomized clinical trials. Arch Gen Psychiatry 59:877-883. doi:10.1001/archpsyc.59.10.877 